\title{
RouX-En-Y Gastric Bypass Improved Intestinal Permeability by Regulating Gut Innate Immunity in Diet-Induced Obese Mice
}

\section{Zhangliu Jin}

Second Xiangya Hospital of Central South University

Kai Chen

Second Xiangya Hospital of Central South University

\section{Zhe Zhou}

Second Xiangya Hospital of Central South University

\section{Weihui Peng}

Second Xiangya Hospital of Central South University

Wei Liu ( $\nabla$ liuweixy@csu.edu.cn )

Second Xiangya Hospital of Central South University

\section{Research Article}

Keywords: Roux-en-Y gastric bypass, Intestinal permeability, Innate immunity, Intestinal alkaline phosphatase, Antimicrobial peptides

Posted Date: December 30th, 2020

DOI: https://doi.org/10.21203/rs.3.rs-132279/v1

License: (c) (1) This work is licensed under a Creative Commons Attribution 4.0 International License. Read Full License

Version of Record: A version of this preprint was published at Scientific Reports on July 21st, 2021. See the published version at https://doi.org/10.1038/s41598-021-94094-8. 


\section{Abstract}

Roux-en-Y gastric bypass (RYGB) has been proven to be the most effective treatment for morbid obesity, yet the impact of RYGB on intestinal permeability is fully unknown. In this work, we subjected obese mice to RYGB and sham operation procedures. Serum lipopolysaccharide (LPS), inflammatory cytokines and intestinal permeability were measured at 8 weeks postsurgery. In contrast to sham surgery, RYGB reduced body weight, improved glucose tolerance and insulin resistance, and decreased serum levels of LPS, IL6 and TNFa. Intestinal permeability of the common limb and colon was significantly improved in the RYGB group compared to the sham group. The mRNA levels of IL1 $\beta$, IL6, and TLR4 of the intestine were significantly decreased in the RYGB group compared with the sham group. The expression of intestinal islet-derived $3 \beta$ (REG3 $\beta$ ), islet-derived $3 \gamma$ (REG3Y) and IAP was higher in the RYGB group than in the sham group. In conclusion, in a diet-induced obesity (DIO) mouse model,RYGB improved intestinal permeability and attenuated systemic inflammation by downregulating intestinal inflammation and innate immunity, which might result from enhanced production of IAP and antimicrobial peptides.

\section{Introduction}

To date, accumulating evidence has suggested that crosstalk between the microbiota and intestine is essential for body weight homeostasis ${ }^{1-3}$. Intestinal bacterial-derived pathogens such as lipopolysaccharide (LPS) play an important role in obesity-related metabolic disorders ${ }^{4}$. A wide range of studies have found that LPS appears to induce chronic low-grade inflammation and regulate fat deposition, which causes insulin resistance and type 2 diabetes mellitus (T2DM) ${ }^{5,6}$.

The gastrointestinal tract confines intestinal bacterial invasion through its physical, chemical and immune barriers, while maintaining proper permeability for the absorption of nutrients ${ }^{7}$. A large number of studies have found impairment of intestinal barrier function in patients with morbid obesity, mainly presenting as increased gut permeability, which might lead to systemic inflammation ${ }^{3,8}$. Moreover, gutderived inflammation exerted a profound influence on intestinal permeability.

Roux-en-Y gastric bypass (RYGB) surgery is the most effective and sustainable treatment for obesity and its comorbidities, such as T2DM and cardiovascular disease ${ }^{9}$. Furthermore, growing body of literature has revealed that the RYGB procedure improves the state of chronic low-grade inflammation in obesity ${ }^{10,11}$. The alimentary tract was realigned after RYGB, which led to fundamental changes in nutrient digestion and absorption, intraluminal environment and intestinal epithelial cells. Meanwhile, intestinal alkaline phosphatase (IAP) and antimicrobial peptides produced by enterocytes play a pivotal role in neutralizing toxicity from intestinal microbiota as part of the innate immune system and subsequently maintaining intestinal homeostasis ${ }^{12,13}$. Hence, we hypothesized that intestinal permeability was improved by RYGB. In this study, we first report the alteration of intestinal permeability in different intestinal limbs after RYGB in a DIO mouse model, with mechanistic investigation from the perspective of intestinal inflammation and innate immunity. 


\section{Methods}

\section{Experimental Animals}

Fourteen male C57BL/6 mice were purchased from SLAC Laboratory Animal Inc. (Shanghai, China) at seven-weeks-old. All animals received water and normal chow (SLAC, Laboratory Animal Inc., Shanghai, China) ad libitum after birth. At the age of 8 weeks, all animals were fed a high-fat diet (HFD) (60 kcal\% fat, \#D12492; Research Diets Inc., New Brunswick, NJ, USA) for 12 weeks and subsequently allocated to two body weight-matched groups (sham and RYGB) before undergoing surgery. All mice were housed under a 12-h light/12-h dark cycle at room temperature $\left(21 \pm 2^{\circ} \mathrm{C}\right)$. The procedures of all animal investigations were performed according to Central South University guidelines for the use of animals, with the approval of the Institutional Animal Care and Use Committee in Central South University. This study was carried out in compliance with the ARRIVE guidelines.

\section{Preoperative Preparation}

Fourteen mice were randomly allocated into two groups: the RYGB group (RYGB surgery, $n=7$ ), and the sham group (sham operation, $n=7$ ). All animals fasted overnight presurgery. The surgical procedures were performed under anesthesia with isoflurane (3-4\% for induction and $1-2 \%$ for maintenance).

\section{Surgery}

All animal surgeries were performed under an approved surgical model protocol ${ }^{14}$. In brief, a 1.0-cm midline incision was made on the upper abdomen. For mice in the RYGB group, the jejunum was transected $4.0 \mathrm{~cm}$ distal to the Treitz ligament, and the proximal part was the biliopancreatic limb. Four centimeters of the distal part was measured and designated as the alimentary limb and then anastomosed to the biliopancreatic limb in an end-to-side fashion by interrupted suture. The gastroesophageal junction was ligated to exclude the stomach, and then a longitudinal incision of $0.2 \mathrm{~cm}$ was made on the distal esophagus. The alimentary limb was anastomosed to the distal esophagus in side-to-side pattern by running sutures. The abdominal incision was closed followed by postoperative care. Mice in the sham group underwent jejunal transection and reanastomosis in situ, and the operating time was extended to be the same as that of the RYGB group.

\section{Postoperative Care}

After surgery, all animals were placed on a heating pad after injection of $1.0 \mathrm{ml} 0.9 \%$ of saline and 1.0 $\mathrm{mg} / \mathrm{kg}$ buprenorphine. Mice received a liquid diet (ENSURE, Abbott, Zwolle, Netherlands) for 24 hours after surgery and then were switched to HFD.

\section{Body Weight, IPGTT, and ITT}

Mouse body weight was measured weekly at 11:00-12:00 a.m to the nearest $0.1 \mathrm{~g}$. After 16 hours of fasting at postoperative week 7, an intraperitoneal glucose tolerance test (IPGTT) was performed after 
administering glucose solution ( $1.5 \mathrm{~g} / \mathrm{kg}$ body weight) by intraperitoneal injection. The insulin tolerance test (ITT) was conducted with intraperitoneal injection of insulin at $0.75 \mathrm{u} / \mathrm{kg}$ body weight after 4 hours of fasting. At $0,15,30,60,90$ and 120 min after injection, mouse blood was retrieved from the tail vein for blood glucose measurement using an ACCU-CHEK Performa glucometer (Roche, Mannheim, Germany).

\section{Plasma IL-6, TNFa and LPS}

Mice were euthanized at 8 weeks postsurgery, followed by the collection of blood and tissue samples. Plasma IL6 and TNFa levels were measured with the relevant enzyme-linked immunosorbent assay kits (BiomeTech, Greifswald, Germany) and plasma LPS concentrations were determined using the limulus amebocyte lysate (LAL) test (Associates of Cape Cod, East Falmouth, MA, USA).

\section{RNA Extraction and Quantitative Real-time PCR}

The intestine of RYGB mice was divided into four segments after RYGB, including the alimentary limb, biliopancreatic limb, common limb and colon, which were rapidly frozen in liquid nitrogen after sampling and then stored at $-80^{\circ} \mathrm{C}$ for further analysis. The common limb of RYGB mice is equal to the lower small bowel of Sham mice. RNA was extracted from snap-frozen specimens with TRIzol reagent (Invitrogen, CA, USA). Reverse transcription of RNA was performed using CDNA synthesis kits (Thermo Fisher Scientific, NY, USA). SYBR green dye was used as a fluorochrome forquantitative real-time PCR (qPCR) in the ViiA7 System (Life Technology). qPCR was conducted to detect the gene expression levels in the intestine.

\section{Ussing Chamber Experiments and Intestinal Permeability Assay of the Common Limb and Colon}

Intestinal permeability was examined 8 weeks after surgery. For the Ussing chamber experiment, in short, it is essential to keep excised intestinal tissue fresh. The intestinal muscle layer was stripped-off and the mucosa was mounted in a Ussing chamber (WPI Instruments, Sarasota, FL, USA) containing $5 \mathrm{ml}$ of circulating oxygenated Krebs buffer $\left(136 \mathrm{mM} \mathrm{NaCl}, 4.3 \mathrm{mM} \mathrm{KCl}, 1.5 \mathrm{mM} \mathrm{CaCl}_{2}, 1.4 \mathrm{mM} \mathrm{MgSO}_{4}, 27 \mathrm{mM}\right.$ $\left.\mathrm{NaHCO}_{3}, 1.6 \mathrm{mM} \mathrm{KH}_{2} \mathrm{PO}_{4}, \mathrm{pH} 7.30\right)^{15}$. The mucosal-to-serosal flux level of dextran conjugated to fluorescein isothiocyanate (dextran-FITC, Sigma, MO, USA) was expressed as the intestinal permeability. After the dextran probe was added, specimens of serosal buffer were retrieved at $0,30,60,90$ and 120 min and then was added with Krebs buffer/glucose. With respect to data analysis, the concentration was estimated in accordance with a standard curve.

\section{Statistical Analysis}

SPSS 21.0 software (SPSS Inc., Chicago, IL, USA) was used to analyze all results. Statistical analysis of the data was performed by Student's t-test or ANOVA. All data are shown as the mean \pm SEM. $P \leq 0.05$ was considered to be statistically significant.

\section{Results}


The body weight of the RYGB group was similar to that of the sham group at the starting point (before the operation). In contrast, the weight loss of mice in the RYGB group was significantly higher than that of mice in the sham group from 1 week to 8 weeks postoperatively (Fig. 1A). According to the plasma glucose measurement, mice in the RYGB group showed significantly improved glucose tolerance (Fig. 1B, 1C) and insulin resistance compared to those in the sham group (Fig. 1D, 1E).

\section{RYGB Surgery Improved Intestinal Permeability}

The intestinal permeability of the common limb (Fig. 2A) and colon (Fig. 2B) was improved in the RYGB group compared to the sham group, according to the Ussing chamber measurement. Although the mRNA expression levels of certain tight junction proteins (TJPs) in the RYGB group showed a tendency of elevation in contrast to those in the sham group, the difference was not significant (Fig. 2C, 2D).

\section{RYGB Surgery Attenuated Systemic and Intestinal Inflammation}

Compared to those in the sham group, the plasma levels of LPS (Fig. 3A), IL6 (Fig. 3B) and TNFa (Fig. 3C) were found to be significantly reduced in the RYGB group囚indicating that endotoxemia and systemic inflammation were prominently alleviated by RYGB. Regarding intestinal inflammation, the mRNA expression levels of IL1 $\beta$ in the common limb (Fig. 3D) and IL6 in the colon (Fig. 3E) were significantly lower in the RYGB group than the sham group, suggesting RYGB attenuated the inflammatory response of the intestine.

\section{RYGB Surgery Regulated Innate Immunity of the Intestine by Suppressing TLR4 Expression}

The mRNA expression levels of Toll-like receptors showed that the expression of TLR4 in the common limb and colon (Fig. 4A, 4B) was significantly reduced in the RYGB group compared to the sham group. Although the mRNA levels of TLR2 and TLR9 in the common limb and colon presented a decreasing trend in RYGB group, the difference did not reach significance.

\section{RYGB Surgery Enhanced the Production of Antimicrobial Peptides and IAP from the Intestine}

We found that the mRNA expression levels of antimicrobial peptides and IAP were augmented after RYGB surgery. In contrast to the sham group, REG3 $\beta$ mRNA levels in the common limb and the colon were remarkably increased in RYGB group (Fig. 5A, 5B), and the REG3y mRNA expression level in the colon was significantly higher in the RYGB group than in the sham group (Fig. 5B). Moreover, the mRNA expression of IAP from both the common limb and colon was markedly enhanced by RYGB (Fig. 5C, 5D).

\section{Discussion}

To our knowledge, this study is the first report on the change in intestinal permeability after RYGB in a DIO mouse model. Our data showed that gut permeability was improved by RYGB in comparison to sham surgery, with marked amelioration of intestinal and systemic inflammation. In mice receiving RYGB surgery, the small bowel was surgically partitioned into three segments, of which the alimentary and 
biliopancreatic limb functioned in the transport of undigested food and digestive juice, respectively ${ }^{16}$. As a result, macronutrient digestion and absorption mainly occurr within the common limb after RYGB, which might lead to profound changes in the intraluminal milieu within the common limb and colon ${ }^{17,18}$. Therefore, our investigation mainly focused on the common limb and colon after RYGB.

In the present study, we showed that RYGB led to significant weight loss and remission of insulin resistance, demonstrating a successful mouse model of RYGB. As chronic inflammation has been proven to play an essential role in insulin resistance and metabolic syndrome ${ }^{19,20}$, we measured inflammatory cytokines in peripheral blood which concluded that RYGB substantially reduced systemic inflammation, as supported by the finding that IL 6 and TNFa were decreased in the RYGB group. Moreover, our study found that serum LPS levels were significantly lower in the RYGB group than in the sham group, suggesting that the intestine became less permeable to gut-derived toxins, which is consistent with the findings of relieved endotoxemia after RYGB in human studies ${ }^{21-23}$.

Intriguingly, several studies ${ }^{24,25}$ revealed that there is an association between increased gut permeability and obesity-related metabolic disorders. Indeed, aggravated gut permeability persistently leads to the development and progression of chronic inflammation, which causes insulin resistance in obesity ${ }^{26}$. Our data revealed that intestinal permeability was decreased in the common limb and colon after RYGB by the Ussing chamber, indicating that RYGB improved intestinal barrier function, which contributed to the amelioration of systemic inflammation and the remission of insulin resistance ${ }^{27,28}$. In addition, we also found that the mRNA levels of TJPs in the common limb and colon were increased after RYGB, although the difference was not significant.

Studies have found excessive inflammation in the gastrointestinal tract of HFD-induced obese mice and patients with morbid obesity ${ }^{3,29}$. Moreover, inflammatory stress may have an impact on the status of gut permeability. IL6, IL1 $\beta$ and TNFa have been widely used to assess the severity of gut inflammation ${ }^{3}$. Our data showed a significant reduction in inflammatory factors, such as IL $1 \beta$ and IL6, in the common limb and colon, respectively. Hence, it could be concluded that gut inflammation was markedly attenuated by RYGB, which led to improvement in intestinal barrier function and amelioration of systemic inflammation.

The crosstalk between the microbiota and gut plays a pivotal role in regulating intestinal function and permeability ${ }^{30}$. A large body of evidence has revealed that a close physiological and pathological connection exists between intestinal innate immunity and gut inflammatory stress ${ }^{31,32}$. As a consequence, to define the mechanism by which RYGB mitigated enteric and systemic inflammation, we assessed the innate immune system of the intestine. Our data demonstrated that the mRNA expression of TLR4 was significantly decreased in the common limb and colon following RYGB, suggesting the intestinal inflammation caused by the LPS-TLR4 pathway was diminished by RYGB.

IAP, a gut brush border enzyme that can dephosphorylate LPS lipid A to detoxify LPS, is secreted into the intestinal lumen by enterocytes, modulates mucosal inflammation via the TLR4 pathway ${ }^{33}$ and 
maintains gut homeostasis ${ }^{34}$. We found that intestinal IAP production was significantly strengthened by RYGB in the common limb and colon, suggesting that the LPS-TLR4 inflammatory pathway might be downregulated through enhanced IAP secretion. Moreover, intestinal epithelial cells can also generate antimicrobial peptides such as REG3 $\beta$ and REG3y, which play a critical role in suppressing gut grampositive bacteria ${ }^{35,36}$. This study revealed that the expression of REG3 $\beta$ and REG3 $\gamma$ was markedly increased in the common limb and colon after RYGB surgery, which may assist inhibiting gut-derived inflammation and improve gut permeability ${ }^{37,38}$. Taken these together, these findings indicate that augmented production of IAP and antimicrobial peptides may exert protective effects against intestinal inflammatory stress after RYGB, which results in the improvement in gut permeability.

\section{Conclusion}

In this study, we first demonstrated that RYGB improved gut permeability in a DIO mouse model and unveiled the association between improved intestinal permeability and enhanced production of IAP and antimicrobial peptides. This study will help to elucidate the mechanism of RYGB and provide a better understanding of the crosstalk between the gut and obesity.

\section{Declarations}

Acknowledge: This study was supported by the National Nature Science Foundation of China (81670481).

Author contributions: Z.J. and K.C. have contributed equally to this work. Z.J. and K.C. conceived and carried out the experiments. Z.Z. and W.P. collected and analyzed data. W.L. conceived the experiments and reviewed the manuscript. All authors were involved in writing the paper and had final approval of the submitted and published versions. W.L. is responsible for this work and all the data in the investigation.

\section{Additional information}

Competing interests: The authors declared no conflict of interests.

Pubulisher's note: Springer Nature remains neutral with regard to jurisdictional claims in published maps and institutional affiliations.

\section{References}

1. Gholizadeh, P. et al. Microbial balance in the intestinal microbiota and its association with diabetes, obesity and allergic disease. Microb. Pathog. 127, 48-55 (2019).

2. Kusumoto, Y. et al. Bile acid binding resin prevents fat accumulation through intestinal microbiota in high-fat diet-induced obesity in mice. Metabolism 71, 1-6 (2017). 
3. Rainone, V. et al. Upregulation of inflammasome activity and increased gut permeability are associated with obesity in children and adolescents. Int. J. Obes. (Lond). 40(6), 1026-1033 (2016).

4. Bernard, A., Ancel, D., Passilly-Degrace, P., Landrier, J.F., Lagrost, L. \& Besnard, P. A chronic LPSinduced low-grade inflammation fails to reproduce in lean mice the impairment of preference for oily solution found in diet-induced obese mice. Biochimie. 159, 112-121 (2019).

5. Hersoug, L.G., Møller, P. \& Loft, S. Role of microbiota-derived lipopolysaccharide in adipose tissue inflammation, adipocyte size and pyroptosis during obesity. Nutr. Res. Rev. 31(2), 153-163 (2018).

6. Lindenberg, F.C.B. et al. Dietary LPS traces influences disease expression of the diet-induced obese mouse. Res. Vet. Sci.123, 195-203 (2019).

7. Briskey, D., Tucker, P., Johnson, D.W. \& Coombes, J.S. The role of the gastrointestinal tract and microbiota on uremic toxins and chronic kidney disease development. Clin. Exp. Nephrol.21(1), 7-15 (2017).

8. Genser, L. et al. Increased jejunal permeability in human obesity is revealed by a lipid challenge and is linked to inflammation and type 2 diabetes. J. Pathol.246(2), 217-230 (2018).

9. Pekkarinen, T., Mustonen, H., Sane, T., Jaser, N., Juuti, A. \& Leivonen, M. Long-Term Effect of Gastric Bypass and Sleeve Gastrectomy on Severe Obesity: Do Preoperative Weight Loss and Binge Eating Behavior Predict the Outcome of Bariatric Surgery? Obes. Surg. 26(9), 2161-2167 (2016).

10. Sjöström, L. et al. Bariatric surgery and long-term cardiovascular events. JAMA.307(1), 56-65 (2012).

11. Rao, S.R. Inflammatory markers and bariatric surgery: a meta-analysis. Inflamm. Res.61(8), 789-807 (2012).

12. Plaeke, P. et al. Effects of intestinal alkaline phosphatase on intestinal barrier function in a cecal ligation and puncture (CLP)-induced mouse model for sepsis. Neurogastroenterol. Motil. 32(3), e13754 (2019).

13. Nakazawa, M. et al. Duodenal expression of antimicrobial peptides in dogs with idiopathic inflammatory bowel disease and intestinal lymphoma. Vet. J.249, 47-52 (2019).

14. Yin, D.P., Boyd, K.L., Williams, P.E., Abumrad, N.N. \& Wasserman, D.H. Mouse Models of Bariatric Surgery. Curr. Protoc. Mouse Biol.2, 295-306 (2012).

15. Kisser, B. et al. The Ussing Chamber Assay to Study Drug Metabolism and Transport in the Human Intestine. Curr. Protoc. Pharmacol.77, 1-7 (2017).

16. Seyfried, F. et al. Roux-en-Y gastric bypass in mice-surgical technique and characterisation. Obes. Surg. 22(7), 1117-1125 (2012).

17. Casajoana, A., Borden, B., Zarabi, S. \& Roslin, M. Conversion of Laparoscopic Roux-en-Y Gastric Bypass (RYGB) to Single Anastomosis Duodenal Switch (SADS). Obes. Surg.29(10), 3412-3413 (2019).

18. Abellan, I. et al. The influence of the percentage of the common limb in weight loss and nutritional alterations after laparoscopic gastric bypass. Surg. Obes. Relat. Dis.10(5), 829-833 (2014). 
19. Ghorpade, D.S. et al. Hepatocyte-secreted DPP4 in obesity promotes adipose inflammation and insulin resistance. Nature 555(7698), 673-677 (2018).

20. Billeter, A.T. et al. Gastric bypass simultaneously improves adipose tissue function and insulindependent type 2 diabetes mellitus. Langenbecks Arch. Surg.402(6), 901-910 (2017).

21. Guo, Y., Liu, C.Q., Liu, G.P., Huang, Z.P. \& Zou, D.J. Roux-en-Y gastric bypass decreases endotoxemia and inflammatory stress in association with improvements in gut permeability in obese diabetic rats. J. Diabetes11(10), 786-793 (2019).

22. Monte, S.V. et al. Reduction in endotoxemia, oxidative and inflammatory stress, and insulin resistance after Roux-en-Y gastric bypass surgery in patients with morbid obesity and type 2 diabetes mellitus. Surgery 151(4), 587-593 (2012).

23. Netto, B.D. et al. Roux-en-Y gastric bypass decreases pro-inflammatory and thrombotic biomarkers in individuals with extreme obesity. Obes. Surg.25(6), 1010-1018 (2015).

24. Robertson, M.D., Pedersen, C., Hinton, P.J., Mendis, A.S.J.R., Cani, P.D. \& Griffin, B.A. Elevated high density lipoprotein cholesterol and low grade systemic inflammation is associated with increased gut permeability in normoglycemic men. Nutr. Metab. Cardiovasc. Dis.28(12), 1296-1303 (2018).

25. Kashihara, H. et al. Duodenal-Jejunal Bypass Maintains Gut Permeability by Suppressing Gut Inflammation. Obes. Surg.29(9), 2745-2749 (2019).

26. Balakumar, M. et al. Improvement in glucose tolerance and insulin sensitivity by probiotic strains of Indian gut origin in high-fat diet-fed C57BL/6J mice. Eur. J. Nutr.57(1), 279-295 (2018).

27. Yang, PJ. et al. Duodenojejunal Bypass Leads to Altered Gut Microbiota and Strengthened Epithelial Barriers in Rats. Obes. Surg.26(7), 1576-1583 (2016).

28. Casselbrant, A., Elias, E., Fändriks, L. \& Wallenius, V. Expression of tight-junction proteins in human proximal small intestinal mucosa before and after Roux-en-Y gastric bypass surgery. Surg. Obes. Relat. Dis. 11(1), 45-53 (2015).

29. Verdam, F.J. et al. Human intestinal microbiota composition is associated with local and systemic inflammation in obesity. Obesity 21(12), 607-615 (2013).

30. Janowitz, C. et al. Disruption of Intestinal Homeostasis and Intestinal Microbiota During Experimental Autoimmune Uveitis. Invest. Ophthalmol. Vis. Sci.60(1), 420-429 (2019).

31. Lin, Y.H. et al. Aryl hydrocarbon receptor agonist indigo protects against obesity-related insulin resistance through modulation of intestinal and metabolic tissue immunity. Int. J. Obes.43(12), 24072421 (2019).

32. Montalban-Arques, A., Chaparro, M., Gisbert, JP. \& Bernardo, D. The Innate Immune System in the Gastrointestinal Tract: Role of Intraepithelial Lymphocytes and Lamina Propria Innate Lymphoid Cells in Intestinal Inflammation. Inflamm. Bowel. Dis.24(8), 1649-1659 (2018).

33. Hwang, S.W., Kim, J.H., Lee, C., Im, J.P. \& Kim, J.S. Intestinal alkaline phosphatase ameliorates experimental colitis via toll-like receptor 4-dependent pathway. Eur. J. Pharmacol.820, 156-166 (2018). 
34. Lassenius, M.I. et al. Intestinal alkaline phosphatase at the crossroad of intestinal health and disease - a putative role in type 1 diabetes. J. Intern. Med.281(6), 586-600 (2017).

35. Muzaki, A.R.B.M. et al. Long-Lived Innate IL-17-Producing $Y / \delta ~ T$ Cells Modulate Antimicrobial Epithelial Host Defense in the Colon. J. Immunol.199(10), 3691-3699 (2017).

36. Li, Z.Y. et al. Intestinal Metrnl released into the gut lumen acts as a local regulator for gut antimicrobial peptides. Acta. Pharmacol. Sin.37(11), 1458-1466 (2016).

37. Loonen, L.M. et al. REG3y-deficient mice have altered mucus distribution and increased mucosal inflammatory responses to the microbiota and enteric pathogens in the ileum. Mucosal. Immunol.7(4), 939-947 (2014).

38. Tremblay, S. et al. Bile Acid Administration Elicits an Intestinal Antimicrobial Program and Reduces the Bacterial Burden in Two Mouse Models of Enteric Infection. Infect. Immun.85(6), e00942-16 (2017).

\section{Figures}

\section{Figure 1}

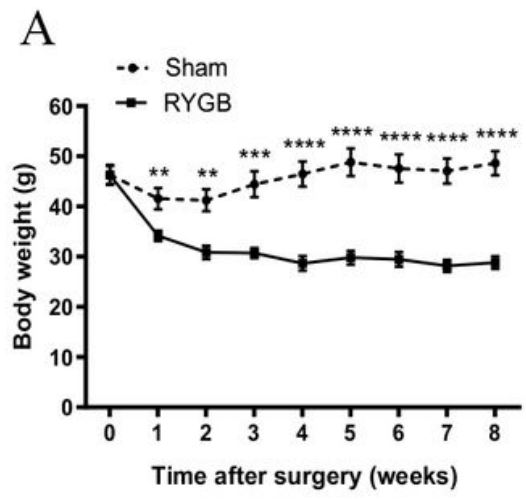

$\mathrm{D}$

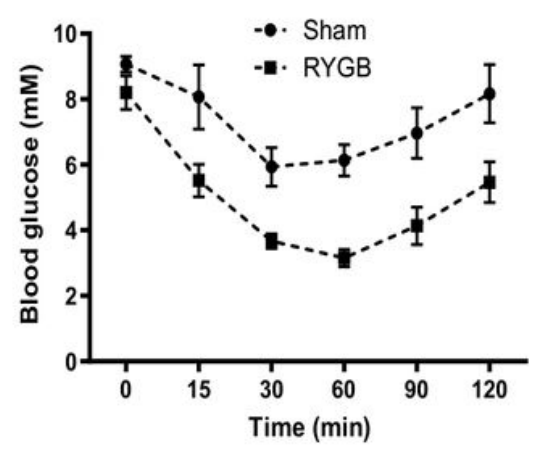

$\mathrm{B}$

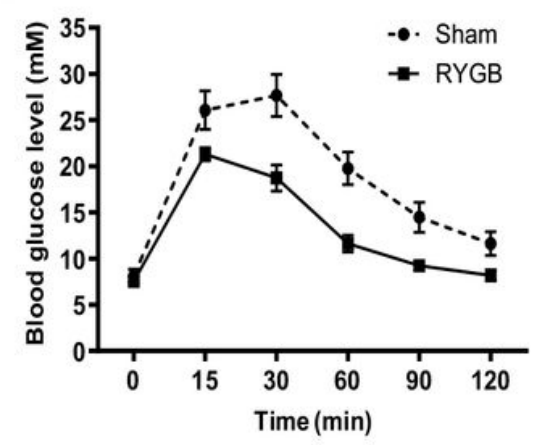

E

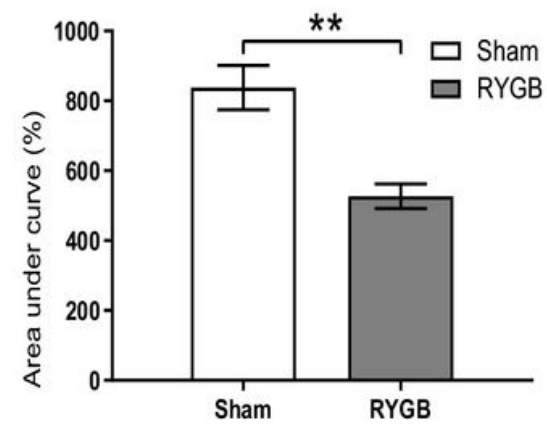

$\mathrm{C}$

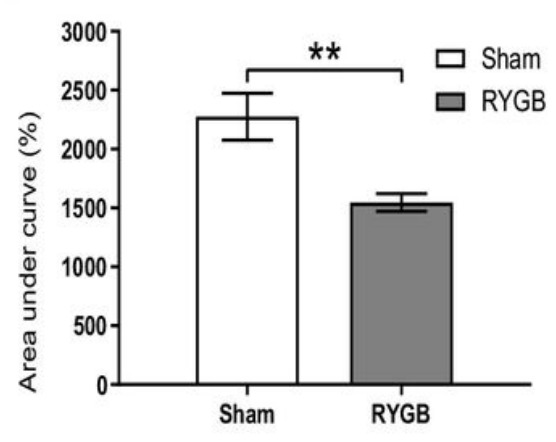

\section{Figure 1}

RYGB reduced body weight and improved glucose homeostasis. (A) The body weight of mice in the RYGB group ( $n=5 /$ group) was significantly lower from postoperative week 1 to week 8 than that of mice in the sham group ( $n=6 /$ group). (B and C) IPGTT measurement and AUC showed that in contrast to sham 
surgery, RYGB significantly reduced glucose levels and improved glucose tolerance. (D and E) The ITT test and AUC revealed that glucose levels were significantly lower in the RYGB group than in the sham group after insulin injection, reflecting an enhanced insulin sensitivity. All data are the mean \pm SEM. ${ }^{*} \mathrm{p}<0.05 ; * * \mathrm{p}<0.01 ; * \star * \mathrm{p}<0.005 ; * \star * * \mathrm{p}<0.001$.

\section{Figure 2}
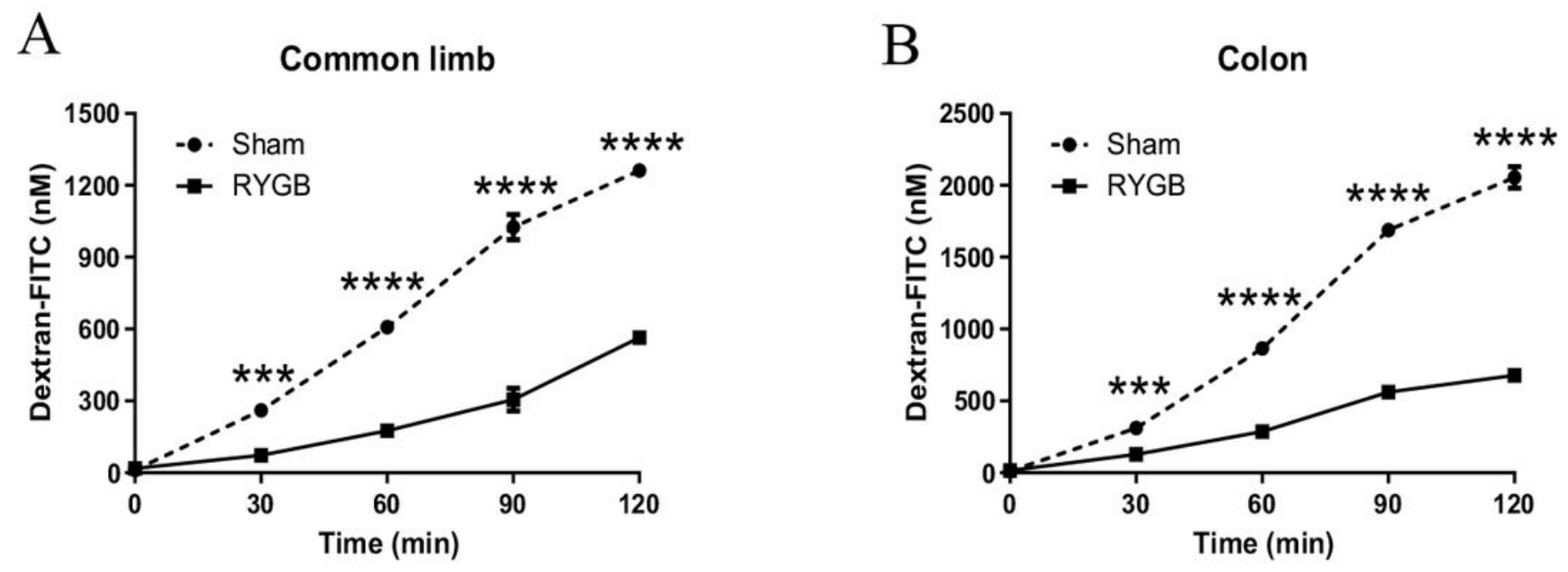

C
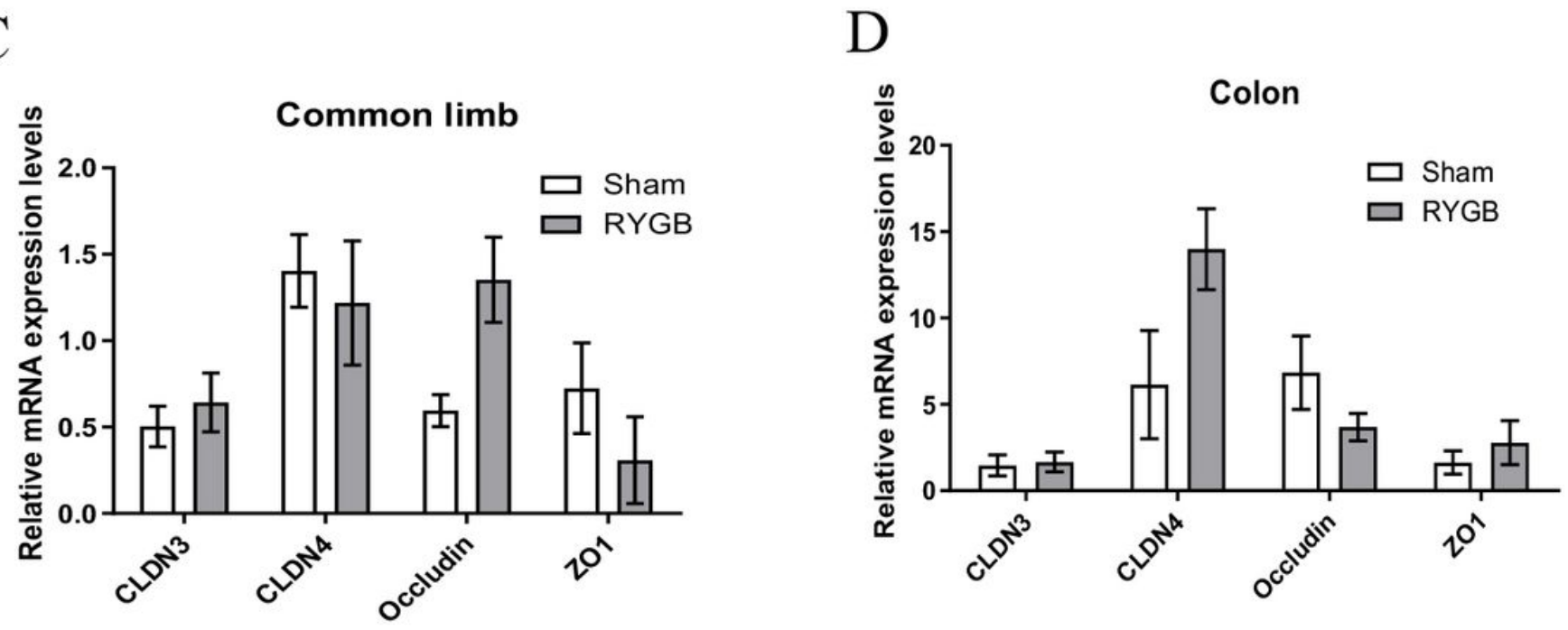

Figure 2

RYGB improved intestinal permeability. Permeability was assessed by the measurement of dextran-FITC in serosal buffer in the Ussing-chamber at $0,30,60,90$, and 120 minutes after the dextran-FITC probe was administered in the mucosal buffer. (A and B) The levels of dextran-FITC in serosal buffer of the common limb and colon were both significantly lower in the RYGB group ( $n=4 /$ group) than in the sham group $(n=4 /$ group). ( $C$ and $D$ ) Measurement of the mRNA expression of TJPs of both the common limb and colon showed no significant difference between the groups, although the RYGB group ( $n=5$ /group) showed a trend of increasing Occludin and CLDN4 in the common limb and colon, respectively, in comparison to in the sham group ( $n=6 /$ group). All data are the mean \pm SEM. CLDN3: claudin-3; CLDN4: claudin-4; Z01: zona occludens-1; ${ }^{*} p<0.05 ;{ }^{* *} \mathrm{p}<0.01 ; * * * \mathrm{p}<0.005 ;{ }^{* * * *} \mathrm{p}<0.001$. 
Figure 3

A

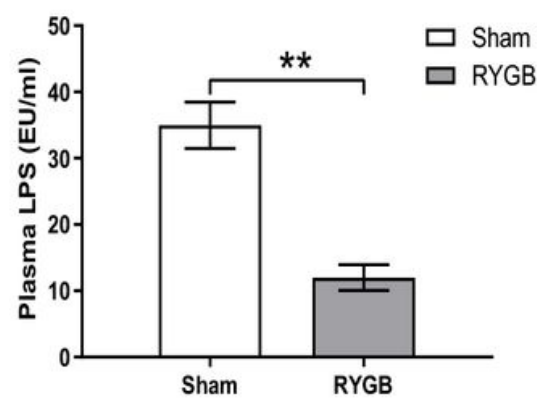

$\mathrm{D}$

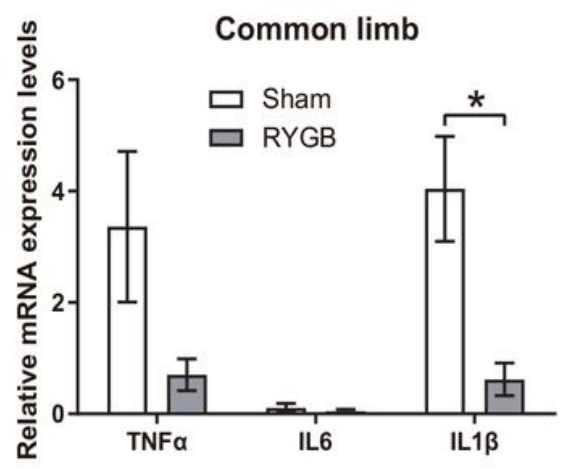

B

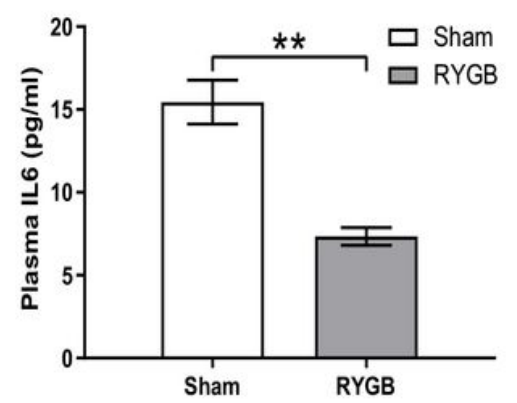

$\mathrm{E}$

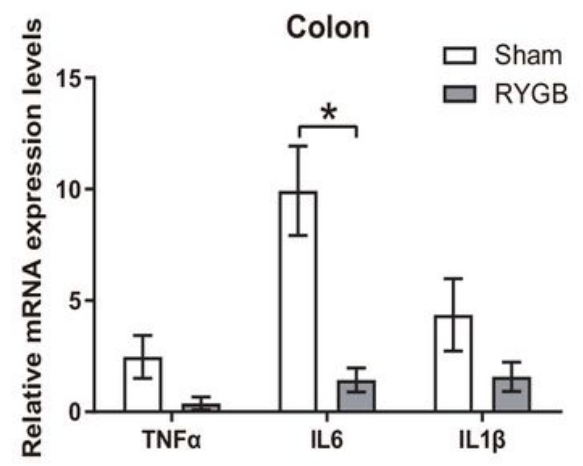

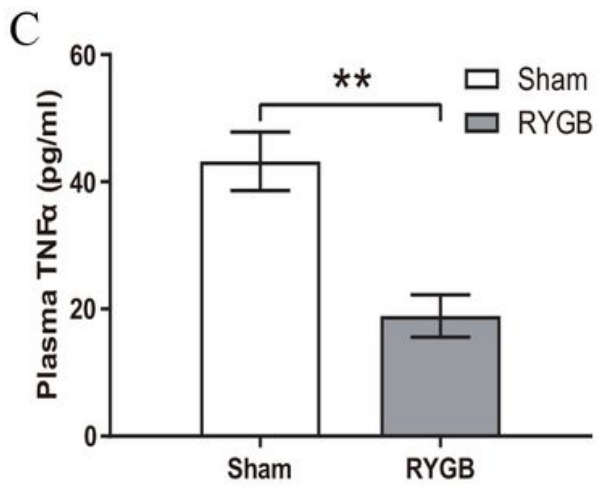

\section{Figure 3}

RYGB suppressed systemic and intestinal inflammation. (A, B and C) Compared to those in the sham group ( $n=6 /$ group), plasma inflammatory cytokines, such as LPS, IL6, and TNFa were notably decreased in the RYGB group ( $n=5 /$ group). $D$ and E: Consistent with these observations, gut inflammation was reduced after RYGB surgery. TNFa, tumor necrosis factor-a; IL6, interleukin-6; IL1 $\beta$, interleukin-1 $\beta$; LPS, lipopolysaccharide. All data are the mean \pm SEM. ${ }^{*} p<0.05 ;{ }^{*} p<0.01$. 
Figure 4

A

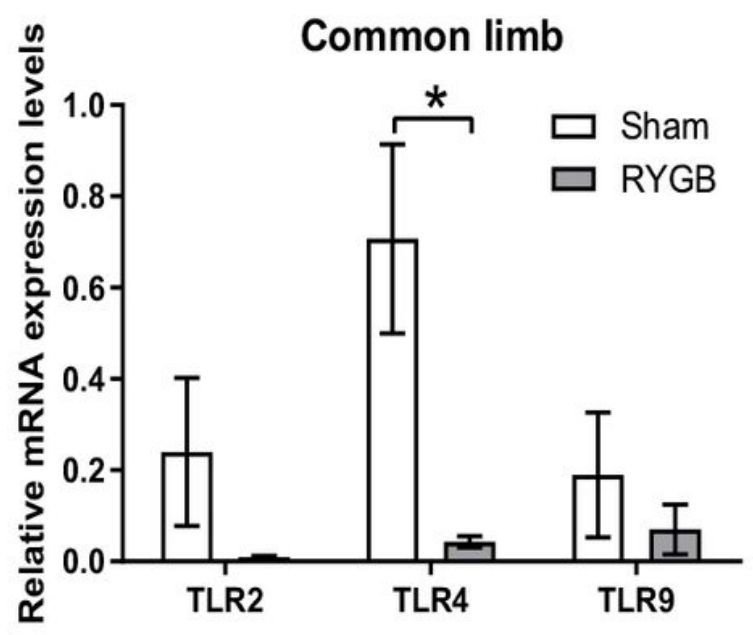

B

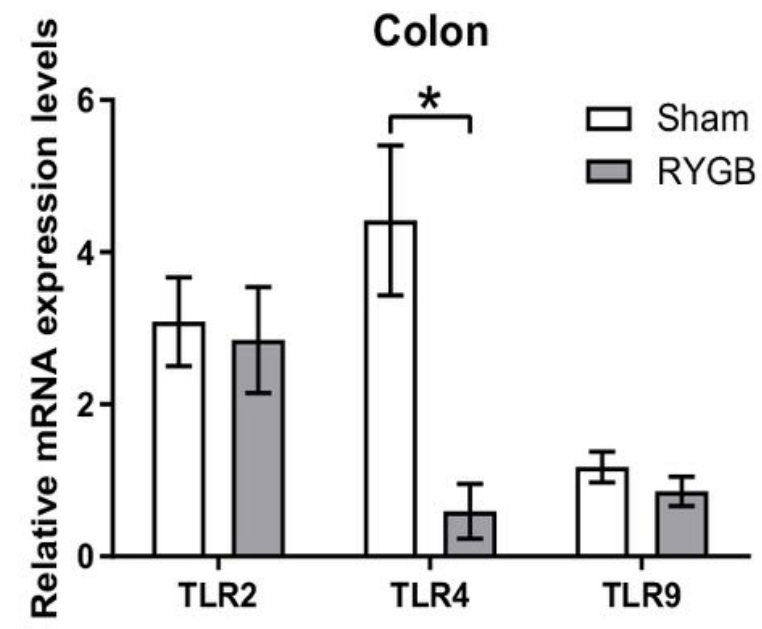

\section{Figure 4}

RYGB regulated the innate immunity of the intestine. ( $A$ and $B$ ) The mRNA expression levels of TLR4 were markedly downregulated in the common limb and colon of mice in the RYGB group ( $n=5 /$ group) compared to the sham group ( $n=6 /$ group). TLR2, Toll-like receptor-2; TLR4, Toll-like receptor-4; TLR9, Tolllike receptor-9. All data are the mean \pm SEM. ${ }^{*} p<0.05$. 
Figure 5

A

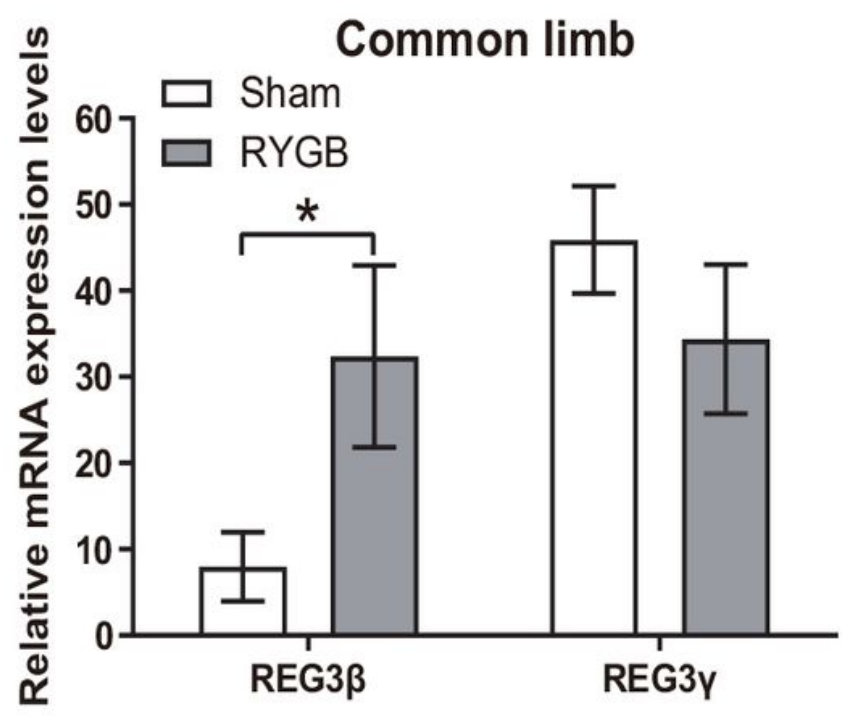

$\mathrm{C}$

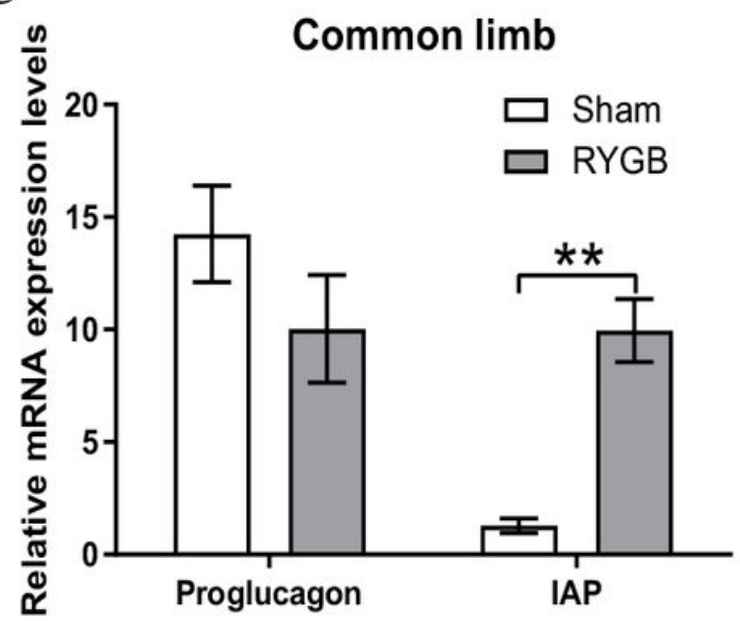

B

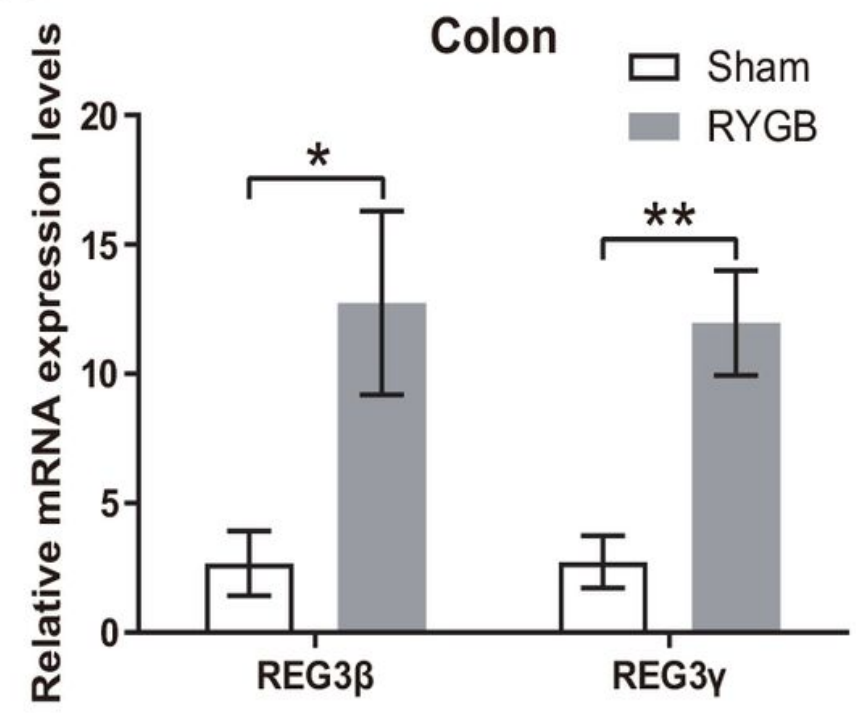

$\mathrm{D}$

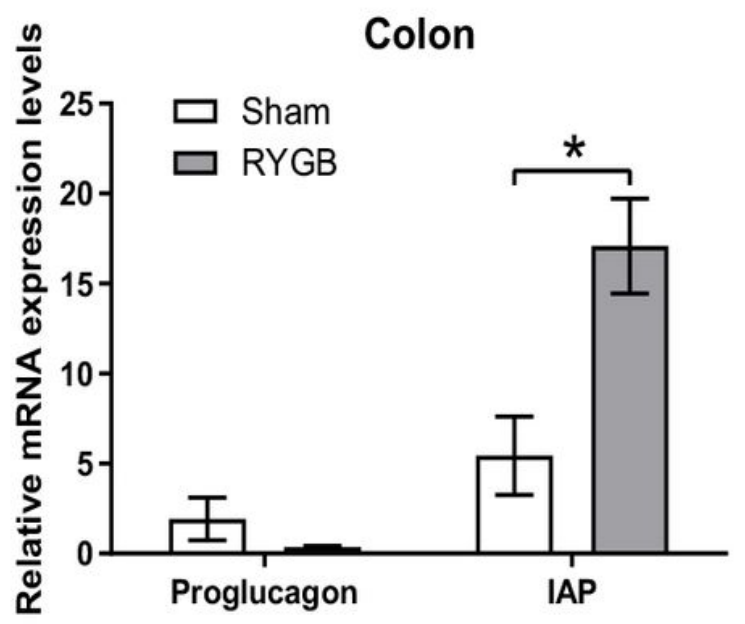

Figure 5

The production of intestinal antimicrobial peptides and IAP was enhanced after RYGB. (A and B) The mRNA expression levels of REG3 $\beta$ were significantly decreased in both the common limb and colon of mice in the RYGB group ( $n=5$ /group) compared to the sham group ( $n=6 /$ group), and the mRNA expression levels of REG3y were reduced in the colon of the RYGB group. ( $C$ and $D$ ) The mRNA expression level of IAP, but not proglucagon, was significantly increased in both the common limb and colon. REG3ß, regenerating islet-derived-3 beta; REG3y, regenerating islet-derived-3 gamma; IAP, intestinal alkaline phosphatase. All data are the mean \pm SEM. ${ }^{*} p<0.05 ;{ }^{*} \mathrm{p}<0.01$. 